\title{
基于裂结技术的单分子化学反应研究进展综述
}

迟力峰

苏州大学功能纳米与软物质研究院, 江苏 苏州 215123

\section{A Review on Recent Progress of Single-Molecule Reaction Investigated by Break Junction Techniques}

\author{
CHI Lifeng \\ Institute of Functional Nano \& Soft Materials, Soochow University, Suzhou 215123, Jiangsu Province, P. R. China. \\ Email: chilf@suda.edu.cn. \\ Published online: March 13, 2019.
}

随着单分子尺度电学表征技术的快速发展, 单 分子电子学的研究已经不再局限于分子电子器件 的研究范畴, 也成为了单分子尺度化学研究的重 要工具。借助单分子尺度电学表征方法, 研究人员 可以将待测分子体系两端修饰诸如硫醇或者吡啶 等针定基团, 通过这些基团与金属电极的相互作 用构筑金属/分子/金属结(分子结), 以此实现对单 个分子的电学性质测量。近年来, 研究人员将单分 子电输运性质的实验测量与理论计算相结合, 从 微观层面理解单个分子的结构与电输运性质之间 的关系, 取得了一系列重要的研究成果, 如单分子 开关 ${ }^{1,2}$ 、单分子二极管 ${ }^{3}$ 的发现等。随着该领域研 究的不断深入, 科学家们尝试将单分子测量技术 与其他表征技术进行联用, 例如与电化学技术 ${ }^{4}$ 、 表面增强拉曼光谱技术联用 5 等, 从而实现电化学 门电压调控、单分子电学-谱学信号同步采集等目 标。同时, 单分子尺度表征技术的精准位移控制和 高灵敏电流测量为研究相互作用体系和化学反应 提供了重要的研究手段。例如光致变色分子化学 反应的动态研究 6 、金属电极上的电场催化研究 7 、 石墨烯电极上主客体之间相互作用的动态平衡研 究 8 等。

近日, 厦门大学化学化工学院洪文晶教授应 邀撰写的综述论文发表于物理化学学报(doi: 10.3866/PKU.WHXB201811027) ${ }^{10}$, 论文总结了国 内外研究人员采用裂结技术在单分子尺度化学反 应研究上取得的一系列重要成果。综述以单分子
裂结技术为出发点展开, 阐述了机械可控裂结技 术(MCBJ)和扫描隧穿裂结技术 (STM-BJ) 的工作 原理, 并对其在单分子尺度化学反应的原位监测、 外场调控、反应动力学等方面的研究进展进行了 综述, 着重介绍电场对化学反应的催化效应及电 化学调控对反应的影响等相关研究工作。

该综述较为全面地概括了单分子裂结技术应 用于研究单分子尺度化学反应这一前沿交叉学科 的最新动态, 对相关领域研究人员具有较高的参 考价值。

\section{References}

(1) Jia, C.; Migliore, A.; Xin, N.; Huang, S.; Wang, J.; Yang, Q.; Wang, S.; Chen, H.; Wang, D.; Feng, B.; et al. Science 2016, 352 (6292), 1443. doi: $10.1126 /$ science.aaf6298

(2) Frisbie, C. D. Science 2016, 352 (6292), 1394. doi: $10.1126 /$ science.aag0827

(3) Guo, C.; Wang, K.; Zerah-Harush, E.; Hamill, J.; Wang, B.; Dubi, Y.; Xu, B. Nat. Chem. 2016, 8, 484. doi: 10.1038/nchem. 2480

(4) O'Driscoll, L. J.; Hamill, J. M.; Grace, I.; Nielsen, B. W.; Almutib, E.; Fu, Y.; Hong, W.; Lambert, C. J.; Jeppesen, J. O. Chem. Sci. 2017, 8 (9), 6123. doi: 10.1039/c7sc02037f

(5) Zheng, J.; Liu, J.; Zhuo, Y.; Li, R.; Jin, X.; Yang, Y.; Chen, Z.; Shi, J.; Xiao, Z.; Hong, W.; et al. Chem. Sci. 2018, 9 (22), 5033. doi: 10.1039/C8SC00727F

(6) Huang, C.; Jevric, M.; Borges, A.; Olsen, S. T.; Hamill, J. M.; Zheng, J.; Yang, Y.; Rudnev, A.; Baghernejad, M.; Broekmann, P.; et al. Nat. 
Commun. 2017, 8, 15436. doi: 10.1038/ncomms 15436

(7) Aragonès, A. C.; Haworth, N. L.; Darwish, N.; Ciampi, S.;

Bloomfield, N. J.; Wallace, G. G.; Diez-Perez, I.; Coote, M. L. Nature 2016, 531, 88. doi: 10.1038/nature16989

(8) Guan, J.; Jia, C.; Li, Y.; Liu, Z.; Wang, J.; Yang, Z.; Gu, C.; Su, D.; Houk, K. N.; Zhang, D. Sci. Adv. 2018, 4 (2), 2177.

doi: $10.1126 /$ sciadv.aar2177
(9) Gu, C.; Hu, C.; Wei, Y.; Lin, D.; Jia, C.; Li, M.; Su, D.; Guan, J.; Xia, A.; Xie, L. Nano Lett. 2018, 18 (7), 4156. doi: 10.1021/acs.nanolett.8b00949

(10) Yu, P.; Feng, A.; Zhao, S.; Wei, J.; Yang, Y.; Shi, J.; Hong, W. Acta Phys. -Chim. Sin. 2019, 35 (8), 829. [余培锴, 冯安妮, 赵世强, 魏珺颖, 杨扬, 师佳, 洪文晶. 物理化学学报, 2019, 35 (8), 829.] doi: 10.3866/pku.Whxb20181102 